\title{
Implicaciones del aprendizaje cooperativo en educación secundaria obligatoria
}

\section{Effects of cooperative learning in secondary compulsory education}

\author{
Pablo Mónico Tamargo, Celestino Rodríguez-Pérez \\ Universidad de Oviedo, España
}

\begin{abstract}
Resumen
El objetivo de este estudio es valorar el impacto de una nueva metodología docente basada en el aprendizaje cooperativo durante la educación secundaria obligatoria. Para ello, se plantea un diseño descriptivo y comparativo con una muestra total de 65 estudiantes y 8 docentes. Los datos recopilados fueron analizados para contrastar las diferencias existentes entre las fases pre-cooperativa y cooperativa. Los resultados muestran que el aprendizaje cooperativo incide positivamente en el clima del aula y el rendimiento académico del alumnado. Sin embargo, no se observan mejoras significativas a nivel de actitudes, comportamiento y motivación de los estudiantes.
\end{abstract}

Palabras clave: motivación, convivencia escolar, rendimiento académico, aprendizaje cooperativo.

\begin{abstract}
The aim of this study is to evaluate the impact of a new teaching method based on cooperative learning in secondary schools. To carry out the study has used a descriptive and comparative design, selecting a sample of 65 students and 8 teachers. All the data collected were analysed in order to contrast the differences between precooperative and cooperative phases. The results shows that cooperative learning has a positive effect on student's academic performance and classroom climate. However, positive consequences related to students' attitudes, behaviour and motivation were not observed.
\end{abstract}

Keywords: motivation, school living together, academic performance, cooperative learning.

La organización escolar se asienta todavía, en muchos casos, sobre principios y procedimientos de trabajo de cohorte clásico caracterizados por el fomento del individualismo en los procesos de aprendizaje, la homogeneidad de la enseñanza y la pasividad en la acción o el carácter reactivo de los agentes educativos. Ante esta concepción, cada vez son más las voces que se alzan con objeto de cambiar la dinámica en las aulas, retomando teorías y pensamientos ya expuestos en otras épocas pero aún no consolidados como debieran.

Las necesidades de la sociedad actual distan de ser las mismas que en el pasado y por eso los procesos de enseñanza-aprendizaje tienden a adaptarse a los cambios promoviendo la cristalización en el alumnado de competencias clave para la vida más allá del mero almacenaje de conocimientos. En esta línea, los profesionales de muchos centros educativos han empezado ya a trabajar en el desarrollo de iniciativas que promuevan la formación de personas abogando por valorar la fuerza y el poder de los grupos de trabajo, la solidaridad, el trabajo colaborativo, el respeto y aprovechamiento de la diversidad... Este es el caldo de cultivo que ha permitido implantar en el aula nuevas metodologías docentes que buscan la mejora continua de la actividad en las aulas como vía para conseguir mejores resultados en el futuro.

El aprendizaje cooperativo es una de esas propuestas metodológicas en boga en nuestros días. Ante esta circunstancia cabe preguntarse ¿Es realmente efectiva esta técnica? ¿Qué tipo de resultados se obtienen con su utilización? ¿Se trata de una buena medida para mejorar la convivencia en las aulas? El interés por ofrecer respuestas a estas y otras cuestiones son las que han motivado el planteamiento de la investigación recogida en el presente trabajo.

A lo largo de los años se ha puesto de manifiesto, desde la literatura y la tradición teórico-práctica la utilidad y los beneficios que genera esta metodología a nivel educativo mediante afirmaciones tales como:

"Las experiencias de aprendizaje cooperativo, comparadas con las [...] competitiva e individualista, favorecen el establecimiento de relaciones entre el alumnado mucho más positivas, caracterizadas por la simpatía, la atención, la cortesía y el respeto mutuo, así como por sentimientos recíprocos de obligación y de ayuda. Estas actitudes positivas se extienden, además, a los profesores y al conjunto de la institución escolar." (Coll, 1984), citado en (Torrego, 2012).

"Las estrategias de enseñanza cooperativa favorece el aprendizaje de todos los alumnos/as, [...] la aceptación de las diferencias entre los estudiantes y la modificación de las relaciones interpersonales en cantidad y calidad." (Parrilla, 1992), citada en (Pujolás, 2004) y (Torrego, 2012).

Recientemente se han llevado a cabo investigaciones en las que se corroboran o demuestran planteamientos y aspectos destacados por las diferentes teorías y corrientes psicopedagógicas que apoyan la potencialidad del aprendizaje cooperativo como la teoría genética de Piaget, la teoría sociocultural de Vygotsky, la teoría de la interdependencia social de David y Roger Johnson, la teoría del aprendizaje significativo de Ausubel o la teoría de las inteligencias múltiples de Gardner entre otras. A consecuencia de los estudios llevados a cabo por diferentes autores interesados en la materia se han obtenido resultados que permiten extraer conclusiones relevantes como las que a continuación se presentan:

"El aprendizaje cooperativo facilita el aprendizaje más que la enseñanza tradicional. [...] Los alumnos/as 
que han seguido el aprendizaje cooperativo logran un mejor resultado en el test final independientemente del modo en que se evalúe. [...] El aprendizaje cooperativo consigue que los estudiantes estén más interesados y motivados durante las clases.” (Méndez, 2012).

"Los contenidos aprendidos mediante técnicas cooperativas planificadas y desarrolladas en el aula son aprendidos y fijados en mayor medida que si se estructuran de modo marcadamente individual. [...] La metodología cooperativa mejora la convivencia y el clima de la clase y el mayor conocimiento entre sus compañeros que no se conocían con anterioridad en casi todos los casos estudiados. [...] El aprendizaje cooperativo permite al alumnado participar más y mejor. El rendimiento académico ha mejorado de un trimestre a otro gracias al cambio de metodología. La mejora en dos tercios del grupo escolar en todos los casos junto a los escasos descensos de calificación del resto de estudiantes que empeoran manifiestan la eficacia del aprendizaje cooperativo frente al tradicional.” (Ruiz, 2012).

"El aprendizaje cooperativo es una metodología extraordinariamente eficaz en algunos aspectos de la enseñanza y, en particular, sobre el efecto que tiene en la convivencia en el aula. Mediante ella, los alumnos/as se conocen más en profundidad y se ven animados a ayudarse mutuamente. Precisamente, esta investigación ha puesto de relieve que los estudiantes acogen este sistema de enseñanza muy positivamente, salvo contadas excepciones, y que los profesores valoran que los estudiantes han aprendido y disfrutado con la misma." (Rodríguez, 2013).

Estas y otras investigaciones han servido para plantear como objetivo del estudio la evaluación del impacto y la eficacia de la introducción de una nueva metodología pedagógica de trabajo en el aula basada en el aprendizaje cooperativo a partir de la identificación de la influencia que ejerce sobre las actitudes, el comportamiento, la convivencia y el rendimiento académico del alumnado.

\section{Método}

El presente trabajo parte de la hipótesis de que la implantación de una nueva metodología de aprendizaje cooperativo en el aula garantiza una mejora de resultados a nivel de actitudes, comportamiento, rendimiento académico, funcionamiento del grupo clase y la satisfacción de estudiantes y profesorado.

Para evaluar los efectos de la metodología docente se ha utilizado un diseño descriptivo y comparativo de tipo longitudinal basado en la recopilación y análisis de datos cuantitativos y cualitativos.

\section{Participantes}

La población objeto de estudio se corresponde con el conjunto del alumnado de $2^{\circ}$ de educación secundaria obligatoria del IES Bernaldo de Quirós (MieresAsturias) y parte del profesorado que imparte docencia en dicho centro y nivel educativo. El total de la muestra asciende a 65 estudiantes con edades comprendidas entre 13 y 15 años, y 8 docentes, cuya media de edad se sitúa en torno a los 42 años. La distribución de la muestra según la variable sexo resultó ser más equilibrada a nivel del alumnado (34 chicos y 31 chicas) que de profesorado (2 hombres y 6 mujeres). Todos ellos accedieron a colaborar voluntariamente con la investigación planteada.

Para facilitar la consecución de los objetivos planteados todos los estudiantes seleccionados fueron distribuidos en tres grupos de estudio en función de su implicación en dinámicas de trabajo basadas en aprendizaje cooperativo.

Tabla 1.

Distribución del alumnado

\begin{tabular}{lcc}
\hline Grupo & Aula & $\mathrm{N}^{\mathrm{a}}$ de estudiantes \\
\hline Cooperativo & $2^{\circ}$ ESO C & 20 \\
No cooperativo & $2^{\circ}$ ESO A & 23 \\
No cooperativo & $2^{\circ}$ ESO B & 22 \\
\hline
\end{tabular}

Asimismo, se optó por potenciar la homogeneidad en el profesorado a nivel de pautas educativas y uso de técnicas cooperativas en el aula, seleccionando, para ello, a aquellos docentes que formaban parte del grupo de trabajo de convivencia e innovación metodológica, pioneros en la puesta en marcha de una experiencia piloto de aprendizaje cooperativo en el centro durante el curso escolar 2013-2014.

\section{Instrumentos}

Para llevar a cabo la investigación se han diseñado y aplicado diferentes recursos "ad hoc" destinados a la recopilación de los datos requeridos para cada una de las variables de estudio planteadas.

Tabla 2.

Variables e instrumentos de recogida de información

\begin{tabular}{|c|c|}
\hline Variables de estudio & Instrumentos \\
\hline $\begin{array}{l}\text { Funcionamiento del } \\
\text { grupo-aula }\end{array}$ & Test sociométrico \\
\hline \multirow{3}{*}{$\begin{array}{l}\text { Actitudes y } \\
\text { comportamiento }\end{array}$} & Entrevistas individuales \\
\hline & Informes de seguimiento \\
\hline & $\begin{array}{c}\text { Libros de registro del equipo } \\
\text { directivo }\end{array}$ \\
\hline Rendimiento académico & Informes de evaluación \\
\hline
\end{tabular}

A través del test sociométrico se ha obtenido información sobre las relaciones que se establecen entre los estudiantes a nivel de afectividad, liderazgo y rechazo. Este test constaba de cuatro ítems con cuatro categorías de respuestas posibles en base a elecciones y rechazos en los ámbitos social y académico. De esta manera los estudiantes debían contestar a cada cuestión planteada indicando a quién preferían o descartaban para compartir tiempo de ocio y tareas en el aula. Las respuestas dadas debían seguir un orden de prioridad en función del grado de afinidad o antipatía hacia las personas elegidas teniendo en cuenta que, en cada caso, podían elegir como máximo a tres estudiantes y que la no elección de ninguno de ellos también era aceptada. 
Las entrevistas individuales planteadas tenían como objetivo conocer la opinión de los participantes acerca del proceso de implantación y desarrollo de la metodología de aprendizaje cooperativo en el aula. El propósito de este planteamiento fue abrir un periodo de conversación y debate en el que las personas entrevistadas mostraran sus opiniones libremente y aportarán información adicional sobre las variables objeto de estudio o cualquier otro aspecto que se considerará relevante para el devenir del mismo.

Por otro lado, la utilización de los informes de seguimiento elaborados por el profesorado permitió obtener datos concretos sobre las valoraciones negativas registradas para cada estudiante en función de la mala conducta mostrada o el desinterés de estos hacia la materia y/o el trabajo en el aula, la falta de material necesario para el seguimiento de las clases, la no realización de las tareas encomendadas, los déficits de atención durante el trabajo en el aula y las interrupciones que lastran el normal funcionamiento del proceso de enseñanza-aprendizaje.

Respecto a los libros de registro del equipo directivo han sido utilizados como herramienta para obtener información sobre las medidas disciplinarias adoptadas con los estudiantes. Se revisaron todas las fichas del alumnado incluidas en ellos, extrayendo, datos sobre las salidas de estudiantes al aula de convivencia, las expulsiones del centro y las amonestaciones impuestas por el profesorado a lo largo del curso.

Finalmente, los informes de evaluación fueron consultados con objeto de recopilar información sobre el nivel de absentismo y éxito académico de los estudiantes de cada uno de los grupos analizados.

\section{Procedimiento}

La recogida de datos se llevó a cabo durante el año académico 2013-2014 tras mantener un primer contacto con el equipo directivo y el orientador del centro en el que se procedió a exponer la intención de la investigación propuesta y las implicaciones del desarrollo de la misma para los diferentes agentes implicados. Una vez obtenido su apoyo se compartió la experiencia a llevar a cabo con los docentes que formaban parte del grupo de trabajo de convivencia e innovación educativa del centro con objeto de asegurar su participación, aclarar las prioridades del estudio, concretar la muestra, adaptar los materiales a utilizar y difundir la iniciativa a toda la comunidad. A partir de ahí se puso en marcha la investigación con la recogida de información de carácter cuantitativo a través de la consulta de los documentos oficiales del centro y la aplicación del test sociométrico en horas de tutoría previamente consensuadas con los tutores de cada grupo. De igual modo se estableció un calendario de entrevistas individualizadas y grupos de trabajo para recabar información cualitativa de interés. Una vez concluida esta fase se procedió a analizar los datos cuantitativos recopilados, sometiendo los resultados a análisis de frecuencias y porcentajes, puntuación media y desviación típica mediante el programa estadístico SPSS 20.0 para Windows. Por su parte, la información cualitativa obtenida se analizó mediante la utilización de la técnica DAFO, metodología de estudio que permitió extraer las debilidades y fortalezas referenciadas por los agentes educativos al respecto de la utilización de la nueva metodología de aprendizaje cooperativo y las amenazas y oportunidades externas que pueden potenciar o complicar su aplicación en las aulas.

\section{Resultados}

Los resultados han sido desglosados en diferentes apartados según las variables objeto de estudio. En cada uno se detalla la información cuantitativa analizada, complementando los datos numéricos aportados con información extraída del análisis cualitativo realizado. Se ha adoptado esta estructura desde el convencimiento de su utilidad para facilitar la interpretación de las conclusiones alcanzadas.

\section{Funcionamiento del grupo-aula}

Durante la implantación y desarrollo de la nueva metodología docente el grupo cooperativo no sufrió alteraciones profundas en cuanto a estructura y relaciones socio-académicas atendiendo a las elecciones y rechazos prioritarios realizados por cada estudiante.

Profundizando sobre los resultados llama la atención la distribución de relaciones entre hombres y mujeres existentes en dicho grupo ya que predominan, tanto a nivel académico como social, las que se producen entre personas del mismo sexo. Además se produce un aumento general de parejas con alta afinidad, lo que, a juicio del profesorado, puede deberse a la consolidación de las preferencias mostradas por el alumnado a principio de curso.

Finalmente, destacar que una de las limitaciones más importantes de este estudio radica en la imposibilidad de establecer mediciones comparativas con los grupos no cooperativos respecto a esta variable al no haberse aplicado en estos el test sociométrico al inicio del curso y carecer, por tanto, de elementos de contraste.

\section{Actitudes y comportamiento}

Medidas disciplinarias. Los resultados que se muestran en la tabla 3 muestran un descenso significativo de las medidas disciplinarias aplicadas en el grupo cooperativo $\left(2^{\circ} \mathrm{C}\right)$ a lo largo del curso académico, fundamentado en un $88 \%$ de mejora a nivel de expulsiones de estudiantes al aula de convivencia y en la no existencia de amonestaciones y expulsiones del centro tal y como ya ocurría en la $1^{\circ}$ evaluación. En contraste con estos datos, es preciso destacar el empeoramiento registrado en uno de los grupos no cooperativo ( $\left.2^{\circ} \mathrm{B}\right)$. En este grupo el número de expulsiones de estudiantes al aula de convivencia y las amonestaciones registradas aumentan un $175 \%$ y un $33 \%$ respectivamente. Por otro lado, es preciso señalar que los datos del grupo no cooperativo de $2^{\circ} \mathrm{A}$ no varían con respecto a los obtenidos en la $1^{\circ}$ evaluación.

En consonancia con los datos resultantes, los agentes educativos del centro corroboran un cambio a nivel de una reducción drástica de comportamientos negativos extremos del alumnado del grupo cooperativo lo que ha facilitado el hecho de no tener que aplicar sanciones 
estrictas. Comentan que la reiteración de las normas en el trabajo específico con el grupo y la unidad en el mantenimiento de la disciplina por parte del profesorado han podido influir también positivamente sobre este aspecto. Sin embargo, son conscientes de que el grupo aún conserva ciertas actitudes y comportamientos negativos que, si bien no exigen la intervención mediante duras medidas disciplinarias, dificultan el correcto desarrollo de la actividad en el aula y la consecución de los objetivos previstos con el grupo.

Tabla 3.

Medidas disciplinarias adoptadas durante el curso

\begin{tabular}{ccccc}
\hline Tipo de medida & Grupo & $1^{\circ}$ & $3^{\circ}$ & $\%$ de \\
\hline & & evaluación & evaluación & mejora \\
\hline \multirow{2}{*}{ Expulsiones al aula } & $2^{\circ} \mathrm{A}$ & 0 & 0 & $0 \%$ \\
de convivencia & $2^{\circ} \mathrm{B}$ & 4 & 11 & $175 \%$ \\
& $2^{\circ} \mathrm{C}$ & 8 & 1 & $-88 \%$ \\
& $2^{\circ} \mathrm{A}$ & 0 & 0 & $0 \%$ \\
Amonestaciones & $2^{\circ} \mathrm{B}$ & 6 & 8 & $33 \%$ \\
& $2^{\circ} \mathrm{C}$ & 1 & 1 & $0 \%$ \\
Expulsiones del & $2^{\circ} \mathrm{A}$ & 0 & 0 & $0 \%$ \\
centro & $2^{\circ} \mathrm{B}$ & 1 & 0 & $-100 \%$ \\
& $2^{\circ} \mathrm{C}$ & 0 & 0 & $0 \%$ \\
\hline
\end{tabular}

Por último, aclarar que los óptimos resultados registrados en $2^{\circ} \mathrm{A}$ se deben a que este grupo está formado por aquellos estudiantes con mayor interés por el estudio y menores problemas de comportamiento.

Valoraciones negativas. Los datos reflejan un empeoramiento en el número de valoraciones negativas registradas por el profesorado en los informes de seguimiento relativos al grupo cooperativo $\left(2^{\circ} \mathrm{C}\right)$. Este hecho se manifiesta a nivel del trabajo en clase, donde se registra un aumento del $129 \%$ en el número de valoraciones negativas, $\mathrm{y}$ en el comportamiento y trabajo en casa donde se produce un aumento de dichas valoraciones en un $13 \%$ y un $10 \%$ respectivamente.

Tabla 4.

Valoraciones negativas registradas por el profesorado

\begin{tabular}{ccccc}
\hline $\begin{array}{c}\text { Tipo de } \\
\text { valoración }\end{array}$ & Grupo & $\begin{array}{c}1^{\circ} \\
\text { evaluación }\end{array}$ & $\begin{array}{c}3^{\circ} \\
\text { evaluación }\end{array}$ & $\begin{array}{c}\% \text { de } \\
\text { cambio }\end{array}$ \\
\hline \multirow{3}{*}{ Comportamiento } & $2^{\circ} \mathrm{A}$ & 14 & 14 & $0 \%$ \\
& $2^{\circ} \mathrm{B}$ & 47 & 44 & $-6 \%$ \\
& $2^{\circ} \mathrm{C}$ & 15 & 17 & $13 \%$ \\
Trabajo en clase & $2^{\circ} \mathrm{A}$ & 7 & 10 & $43 \%$ \\
& $2^{\circ} \mathrm{B}$ & 52 & 60 & $15 \%$ \\
& $2^{\circ} \mathrm{C}$ & 14 & 32 & $129 \%$ \\
& $2^{\circ} \mathrm{A}$ & 15 & 14 & $-7 \%$ \\
Trabajo en casa & $2^{\circ} \mathrm{B}$ & 79 & 72 & $-9 \%$ \\
& $2^{\circ} \mathrm{C}$ & 48 & 53 & $10 \%$ \\
\hline
\end{tabular}

Los malos resultados obtenidos por este grupo a nivel de trabajo en casa y comportamiento contrastan con la mejora o el mantenimiento de valoraciones negativas registradas por los grupos no cooperativos en estos ámbitos. Sin embargo, es justo convenir que en cuanto al trabajo en clase todos los grupos objeto de estudio muestran un empeoramiento significativo en la $2^{\circ}$ evaluación.

Estos resultados vienen a corroborar los comentarios transmitidos por el profesorado sobre la existencia de actitudes y comportamientos por parte del alumnado que dificultan el trabajo con el grupo sobre todo a nivel de implicación con las tareas a desarrollar en el aula. Sin embargo, llegados a este punto es también preciso aclarar que algunos miembros del equipo docente consideran que el nivel de empeoramiento no ha sido tan radical sino que el alumnado se comporta, más o menos, como en la $1^{\circ}$ evaluación, salvo por diferencias que achacan al nivel de cansancio acumulado del alumnado y el profesorado. Ante estas explicaciones cabe decir que si tomamos como referencia el cansancio acumulado por los integrantes de los distintos grupos este debería tener efecto en los resultados generales de todos ellos y este hecho no se refleja en los datos extraídos durante la investigación con los grupos no cooperativos. Por tanto, no parece que este aspecto sea el culpable de las diferencias registradas en los grupos. Si bien, existen otros que, en palabras de los docentes, si pudieran tener más influencia como el hecho de no contar con protocolos estrictos de asignación de valoraciones negativas para el profesorado. Esto provoca que cada uno, de manera subjetiva, valore cuando es conveniente o no dicha asignación y su correspondiente registro en los informes de seguimiento. En opinión del profesorado este fenómeno tiene menor incidencia a nivel de medidas disciplinarias pues en el caso de estas existen ciertas pautas que permiten unificar criterios con respecto a su aplicación.

\section{Rendimiento académico}

Como se puede observar en la tabla 5, el grupo objeto de estudio $\left(2^{\circ} \mathrm{C}\right)$ es el único que consigue una mejora significativa a nivel de resultados académicos en la tercera evaluación, cifrándose esta mejora en un $17,37 \%$. Este dato se explica por el incremento de estudiantes que aprueban todo o son susceptibles de promoción al curso siguiente. En este caso, el alumnado de $2^{\circ} \mathrm{A}$ y $2^{\circ} \mathrm{B}$ (grupos no cooperativos) empeoran sus resultados. Esto es evidente en el caso de $2^{\circ} \mathrm{B}$ que desciende 4,55 puntos porcentuales con respecto al resultado satisfactorio obtenido en la $1^{\circ}$ evaluación. En lo referente al $2^{\circ} \mathrm{A}$ parece que a priori los resultados se mantienen pero al bajar al detalle de los mismos se observa que a pesar de mantener un $100 \%$ de resultado satisfactorio el número de estudiantes que aprueban todo en tercera evaluación desciende por lo que, en cierta manera, el nivel académico del grupo empeora sensiblemente. 


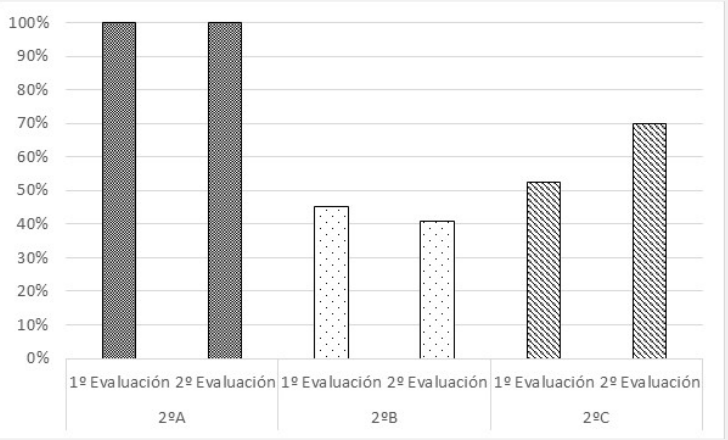

Figura 1. Rendimiento académico del alumnado

Las reacciones observadas en el profesorado y los comentarios recogidos tanto en las sesiones de evaluación como en las reuniones de tutores y equipos docentes ponen de manifiesto su satisfacción por los resultados obtenidos por el grupo piloto. Afirman que el trabajo con el alumnado de este grupo durante el curso evaluación ha evolucionado en productividad y consideran que aunque la mejora puede deberse a la utilización de la nueva metodología de aprendizaje cooperativo en el aula existen también otros factores que han podido ejercer influencia sobre la misma resaltando, como el más relevante, la unidad y coordinación mostrada por el profesorado a la hora de trabajar con el grupo y aplicar medidas correctoras en el mismo.

\section{Valoraciones globales y grado de satisfacción de los agentes implicados}

Como ya se ha comentado previamente, durante el transcurso de la investigación, se procedió a recabar datos de carácter cualitativo sobre las implicaciones del aprendizaje cooperativo en el aula a través de diversas entrevistas y grupos de trabajo. El resultado de este proceso fue analizado a través de la técnica DAFO extrayendo conclusiones que permiten conocer las bondades y limitaciones de la metodología así como las dificultades y facilidades encontradas para su implantación en las aulas.

Debilidades. Entre los puntos débiles de la iniciativa destacan la inexistencia de un protocolo de implantación y seguimiento del trabajo cooperativo en el aula, la ausencia de recursos específicos para la aplicación de la nueva metodología docente, el seguimiento parcial de la medida y la falta de interés mostrado por algunos profesionales del centro.

Amenazas. Entre los factores cuya influencia debe ser atenuada destacan la exigencia sobre la impartición de la totalidad de contenidos del currículo, el nivel de burocracia actual al que se ven sometidos los docentes, la falta de compromiso del alumnado y la incomprensión o las dudas mostradas por algunos profesionales sobre la utilidad de la metodología cooperativa.

Fortalezas. Entre los puntos fuertes de la medida destacan un aumento de la responsabilidad y la ayuda mutua entre estudiantes, una mayor integración del alumnado en el aula, un incremento de la motivación inicial del alumnado para afrontar las tareas académicas y la mejora del clima del aula.

Oportunidades. Entre los factores a aprovechar en un futuro cabe destacar la actual sensibilización del profesorado hacia la búsqueda de la excelencia educativa y la implantación de métodos innovadores durante el proceso de enseñanza-aprendizaje en las aulas, la proliferación de cursos, talleres y grupos de trabajo sobre aprendizaje cooperativo, la experiencia de otros centros y profesionales en la implantación de esta metodología y la disponibilidad de contenidos teóricos y bancos de recursos en la red (foros, blogs, webs educativas..).

En líneas generales tanto el alumnado del grupo cooperativo como el profesorado que imparte docencia en el mismo han mostrado un alto grado de satisfacción con la nueva metodología de trabajo en el aula, valorando especialmente los efectos positivos del cambio adoptado a nivel del funcionamiento y el clima general del aula.

\section{Discusión}

La pretensión del trabajo realizado nuca fue otra que la de servir de apoyo a la futura toma de decisiones por parte del grupo de trabajo de convivencia del centro sobre las medidas a desarrollar durante los próximos cursos académicos.

En virtud de los datos extraídos y comentados hasta este punto se puede concluir que la implantación de una metodología de trabajo cooperativo en el aula ejerce una influencia positiva sobre muchos de los aspectos analizados aunque sus efectos no abarquen la totalidad de mejoras pretendidas. Así, tal y como se observa en los resultados obtenidos, dicha metodología incide positivamente en:

a) El funcionamiento del grupo clase: ya que aunque no se hayan producido grandes cambios a nivel de estructura del grupo y dinámica de relaciones en este caso concreto, si es cierto que se han observado mejoras relacionadas con el clima del aula por ejemplo a través de una mayor solidaridad del alumnado y un aumento en el número de relaciones de afinidad del grupo.

b) El rendimiento escolar: puesto que los resultados obtenidos por el alumnado tras la implantación de la nueva metodología superan a aquellos registrados en las aulas en las que se sigue una metodología de carácter más tradicional.

c) La valoración y satisfacción mostrada por el alumnado y el profesorado ha sido también positiva puesto que ambos colectivos han otorgado puntuaciones altas a la utilidad de la iniciativa.

Sin embargo, a tenor de lo expuesto, no se puede concluir que la influencia del aprendizaje cooperativo redunde en una mejoría a nivel de:

a) Actitudes y comportamiento del alumnado: los datos obtenidos reflejan que el número de valoraciones negativas y quejas del profesorado sobre el alumnado del grupo cooperativo lejos de reducirse fue progresivamente en aumento durante el curso.

b) Motivación del alumnado: el interés mostrado por el alumnado hacia las materias y los contenidos 
impartidos en ellas no ha variado con la implantación de la nueva metodología de ahí que también se resienta el trabajo realizado en clase y la consecución de las tareas de estudio a realizar fuera del centro.

En definitiva, en base a lo dicho, es preciso añadir que tanto la hipótesis de partida como muchas de las conclusiones alcanzadas por los investigadores que han sido tomados como referentes al inicio del proyecto no se cumplen, al no haberse contrastado, en este caso, un nivel de mejora significativa en el ámbito de la motivación, actitudes y comportamiento mostrado por el alumnado del grupo cooperativo. Con todo, aquellas otras conclusiones extraídas de las investigaciones comentadas en referencia a los efectos positivos de esta metodología sobre el rendimiento y la convivencia han sido ratificadas con el estudio.

En base a todo ello, se considera oportuno continuar con la iniciativa durante el próximo curso académico ampliando la experiencia a un mayor número de grupos en el centro, potenciando las fortalezas identificadas, subsanando las debilidades detectadas y favoreciendo su complementariedad con otras medidas de carácter metodológico y organizativo. Sería conveniente, además, continuar la investigación realizada en los años académicos venideros, solventando las limitaciones del presente estudio con objeto de contrastar los resultados aquí descritos. Además, resultaría interesante poder ampliar el estudio para cotejar el impacto de iniciativas similares a la analizada en otros centros y etapas educativas. Ambas propuestas redundarían en la posibilidad de realizar análisis comparativos a partir de los cuales obtener resultados más globales que potencien su utilidad científica como referencia para otros casos en los que se esté planteando la adopción de metodologías de aprendizaje cooperativo en las aulas.

\section{Referencias}

Camilli, C., López, E. \& Barceló, M.L. (2012). Eficacia del aprendizaje cooperativo en comparación con situaciones competitivas o individuales. Su aplicación en la tecnología: una revisión sistemática. Enseñanza \& Teaching, 30, 2-2012, 81-103. http://campus.usal.es/ revistas_trabajo/index.php/021 2-5374/article/viewFile/9316/9609

Coll, C. (1984). Estructura grupal, interacción entre alumnos y aprendizaje escolar. Infancia y aprendizaje, 27/28, 119-138.

http://dialnet.unirioja.es/servlet/articulo?codigo=6684 49

Goicoetxea, E. \& Pascual, G. (2002). Aprendizaje cooperativo: bases teóricas y hallazgos empíricos que explican su eficacia. Educación XXI, 5, 2002, 199226. http://dx.doi.org/10.5944/educxx1.5.1.392

González, N. \& García, M.L. (2007). El aprendizaje cooperativo como estrategia de EnseñanzaAprendizaje en Psicopedagogía (UC): repercusiones y valoraciones de los estudiantes. Revista Iberoamericana de Educación, 42, 6. http://www.rieoei.org/expe/1723Fernandez.pdf

Grañeras, M. \& Parras, A. (Coord.) (2008) Orientación educativa: fundamentos teóricos, modelos institucionales y nuevas perspectivas. Ministerio de Educación, Política Social y Deporte - CIDE.

León del Barco, B., Felipe, E., Iglesias, D. \& Marugán, M. (2014). Determinantes de la eficacia del aprendizaje cooperativo. Una experiencia en el EESS. Revista de investigación Educativa, 32, 2, 411-424. http://dx.doi.org/10.6018/rie.32.2.172721

Mahmood, N. \& Ahmad, Z. (2010). Effects of Cooperative Learning vs. Traditional Instruction on Prospective Techers' Learning Experience and Achievement. Ankara University, Journal of Faculty of Educational Sciences, 43, 1, 151-164. http://papers.ssrn.com/sol3/papers.cfm?abstract_id=2 361353

Méndez, D. (2012). El aprendizaje cooperativo y la enseñanza tradicional en el aprendizaje de la física. Educación y futuro, 27, 179-200.

Poveda, P. (2006). Implicaciones del aprendizaje de tipo cooperativo en las relaciones interpersonales $y$ en el rendimiento académico. Universidad de Alicante, España. http://rua.ua.es/dspace/bitstream/10045/4110/1/tesis_d octoral_patricia_poveda.pdf

Pujolás, P. (2004). Aprender juntos, alumnos diferentes. Los equipos de aprendizaje cooperativo en el aula. Barcelona: Eumo-Octaedro.

Rodríguez, M. (2013). El aprendizaje cooperativo en el aula de Lengua y Literatura en $3^{\circ}$ de la ESO. Trabajo fin de máster, Facultad de Educación. Universidad Internacional de la Rioja.

Ruiz, D. (2012). La influencia del trabajo cooperativo en el aprendizaje del área de economía en la enseñanza secundaria. Tesis doctoral, Facultad de Educación y trabajo social. Universidad de Valladolid. http://uvadoc.uva.es/handle/10324/2729

Slavin, R. (2014). Cooperative Learning and Academic Achievement: Why Does Groupwork Work? Anales de Psicología, 30, 3, 785-791. http://dx.doi.org/10.6018/analesps.30.3.201201

Torrego, J.C. \& Negro, A. (2012). Aprendizaje cooperativo en las aulas. Madrid: Alianza Editorial.

Trujillo, F. \& Ariza, M.A. (2006). Experiencias educativas en Aprendizaje Cooperativo. Granada: Grupo editorial universitario.

Velázquez, C. (2013). Análisis de la implementación del aprendizaje cooperativo durante la escolarización obligatoria en el área de educación física, Departamento de didáctica de la expresión musical, plástica y corporal, Universidad de Valladolid, España. http://uvadoc.uva.es/handle/10324/2823

\section{Agradecimientos}

Los autores desean mostrar su agradecimiento a todas aquellas personas que han hecho posible este estudio: Equipo directivo, orientador y profesorado del Instituto Bernaldo de Quirós de Mieres (Asturias). Muchas gracias a todos por su colaboración y sus valiosas aportaciones. 